\title{
Tumorigenic transformation by CPI-17 through inhibition of a merlin phosphatase
}

\author{
Hongchuan $\operatorname{Jin}^{1,2} \dagger$, Tobias Sperka ${ }^{1,2}$, Peter Herrlich ${ }^{1,2} \&$ Helen Morrison ${ }^{1,2}$
}

The tumour suppressor protein merlin (encoded by the neurofibromatosis type 2 gene NF2) is an important regulator of proliferation in many cell and tissue types ${ }^{1-4}$. Merlin is activated by dephosphorylation at serine 518 (S518), which occurs on serum withdrawal or on cell-cell or cell-matrix $\operatorname{contact}^{5,6}$. However, the relevant phosphatase that activates merlin's tumour suppressor function is unknown. Here we identify this enzyme as the myosin phosphatase (MYPT-1-PP1 $)$ ). The cellular MYPT-1-PP1 $\delta$-specific inhibitor CPI-17 causes a loss of merlin function characterized by merlin phosphorylation, Ras activation and transformation. Constitutively active merlin (S518A) reverses CPI-17-induced transformation, showing that merlin is the decisive substrate of MYPT-1-PP1 $\delta$ in tumour suppression. In addition we show that CPI-17 levels are raised in several human tumour cell lines and that the downregulation of CPI-17 induces merlin dephosphorylation, inhibits Ras activation and abolishes the transformed phenotype. MYPT-1-PP1 $\delta$ and its substrate merlin are part of a previously undescribed tumour suppressor cascade that can be hindered in two ways, by mutation of the NF2 gene and by upregulation of the oncoprotein CPI-17.

We tested whether carboxy-terminal merlin (amino-acid residues 299-595; C-merlin) carrying the decisive phosphorylation site S518 (ref. 6) could be dephosphorylated and could therefore be used as a substrate in vitro. C-merlin expressed in rat schwannoma RT4 cells under a doxycycline-inducible promoter (RT4tet-C-merlin) ${ }^{5}$ migrated as a distinct doublet. The species with decreased mobility was phosphorylated and converted by treatment with calf intestinal phosphatase to a faster-migrating dephosphorylated band (Fig. 1a) with the same mobility as the non-phosphorylatable S518A mutant protein (in which S518 has been replaced with alanine, in contrast with the pseudo-phosphorylated S518D mutant protein containing an aspartic residue; Fig. 1d). The dephosphorylation of C-merlin was regulated by the same cues as that of full-length merlin $^{5}$, for example, high cell density (Fig. 1b), the addition of high-molecular-mass hyaluronan $(\mathrm{HA})^{5}$ or serum withdrawal (as shown in Fig. 1c by the change in the ratio of the upper to the lower band). Because dephosphorylation took less than 5 min after hyaluronan addition or serum withdrawal, we assumed that the activation of a phosphatase was involved rather than a change in stability of the phosphorylated band.

To search for the phosphatase that activates merlin's tumour suppressor function, we tested several phosphatase inhibitors for their effects on merlin dephosphorylation. Merlin dephosphorylation induced by hyaluronan addition (not shown) or serum withdrawal was inhibited by high but not low concentration of okadaic acid (C-merlin in Fig. 1e; full-length merlin in Fig. 1f; merlin phosphorylation status was detected by a phospho-S518 antibody because full-length merlin resolves less well into two bands). Okadaic acid inhibits the protein serine/threonine phosphatase PP2A at nanomolar concentrations, whereas PP1 is blocked at 10-100-fold higher concentrations ${ }^{7}$. The inhibition characteristics indicated that a PP1-type enzyme might be responsible for merlin dephosphorylation. In an in vitro assay, immunoprecipitated partly phosphorylated C-merlin was indeed completely dephosphorylated by 0.05 units of PP1 but not by 0.05 units of PP2A (Fig. 1g).

Although the catalytic process is fast and transient, enzymes sometimes interact with their substrates relatively stably ${ }^{7,8}$. Indeed, PP1 was co-precipitated with endogenous merlin (Fig. 1h), whereas PP2A could not be detected. PP1 proteins accept many substrates, with specificity being accomplished by specific targeting subunits. We considered it likely that the co-precipitation of PP1 with merlin was mediated by one of these targeting subunits. A prominent band

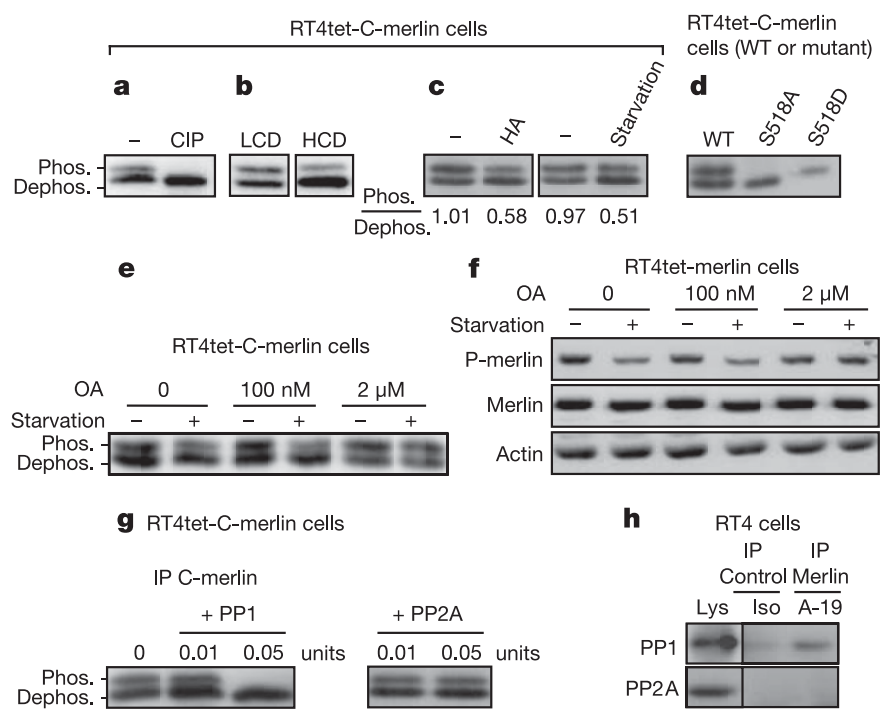

Figure 1 | PP1 is the catalytic subunit of merlin phosphatase.

a, Immunoprecipitated C-merlin was incubated with or without calf intestinal phosphatase (CIP), resolved by SDS-PAGE and detected by immunoblotting (C-18, merlin antibody). b, Immunoblots of C-merlin from cells growing at low cell density (LCD) or high cell density (HCD). c, Immunoblots of C-merlin from cells subjected to serum withdrawal ( $5 \mathrm{~min}$ ) or incubation with hyaluronan (HA; $100 \mu \mathrm{g} \mathrm{ml}^{-1}$ for $5 \mathrm{~min}$ ). d, Gel migration of mutant (S518A, S518D) C-merlin relative to that of the wild type (WT). e, f, Serum-withdrawal-induced dephosphorylation of C-merlin (e) and of full-length merlin probed with phospho-merlin-specific antibodies (f) is inhibited by pretreatment of cells with okadaic acid (OA). g, In vitro dephosphorylation of immunoprecipitated (IP) C-merlin by recombinant PP1 and PP2A. h, Immunoprecipitation (IP) of endogenous merlin (merlin antibody A-19 compared to an isotope control antibody iso). Co-precipitated proteins detected with pan PP1 and PP2A antibodies.

${ }^{1}$ Leibniz Institute of Age Research-Fritz-Lipmann-Institute, Beutenbergstrasse 11, 07745 Jena, Germany. ${ }^{2}$ Forschungszentrum Karlsruhe, Institute of Toxicology and Genetics, PO Box 3640, 76021 Karlsruhe, Germany. †Present address: Cancer Center, Department of Clinical Oncology, Prince of Wales Hospital, The Chinese University of Hong Kong, Hong Kong SAR, China. 
co-immunoprecipitating with $\mathrm{C}$-merlin was identified by mass spectrometry analysis as myosin (myosin heavy chain; Supplementary Fig. S1a). Although a direct interaction between merlin and myosin is possible, we considered a complex with other partners to be more likely. Myosin is known to bind to a phosphatase-targeting subunit termed MYPT-1 (myosin phosphatase targeting subunit 1$)^{9,10}$. Moreover, moesin, a protein with significant sequence homology with merlin, had previously been shown to associate with MYPT-1 (ref. 8). Taken together, these results indicated that MYPT-1 might be the merlin-targeting subunit for PP1. The Coomassie-stained gel also revealed a band at a molecular mass of about $130 \mathrm{kDa}$, which by immunoblot was identified as MYPT-1 (Supplementary Fig. S1a, b).

MYPT-1 prefers PP1 $\delta$ as the catalytic subunit ${ }^{10}$. Indeed, PP1 $\delta$ and MYPT-1 were co-precipitated with endogenous merlin from lysates of both RT4 and NIH 3T3 cells (Fig. 2a). A bacterially expressed glutathione $S$-transferase (GST) fusion of the C-terminal half of MYPT-1 pulled down recombinant $\mathrm{C}$-merlin in vitro, proving a direct interaction between MYPT-1 and merlin (Fig. 2b). Although bound to the non-phosphorylated bacterially expressed C-merlin, MYPT-1 preferred the phosphorylated form of merlin, because fulllength S518D or wild type merlin compared to S518A merlin coprecipitated with MYPT-1 (Fig. 2c and Supplementary Fig. S2). In addition to the direct interaction of MYPT-1 with merlin we showed that an immunoprecipitated MYPT-1-PP1 $\delta$ complex accepts phospho-merlin as a substrate in vitro (Fig. $2 \mathrm{~d}$ ). To determine whether MYPT-1 targets PP1 $\delta$ to merlin or increases the specific activity of

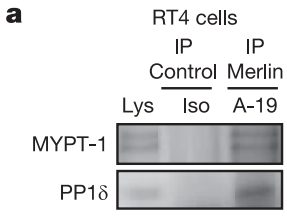

b
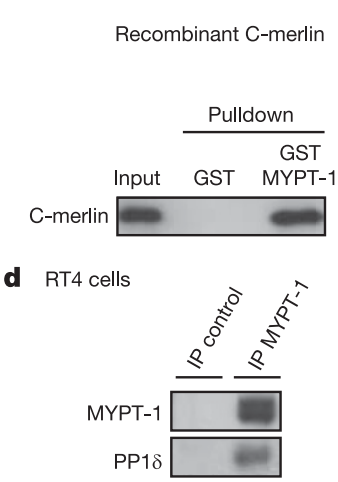

e

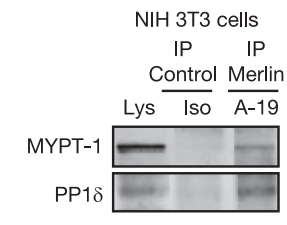

c

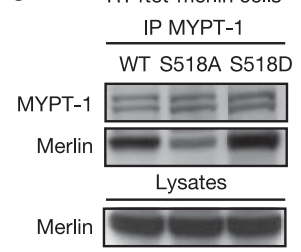

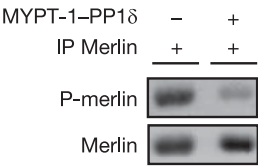
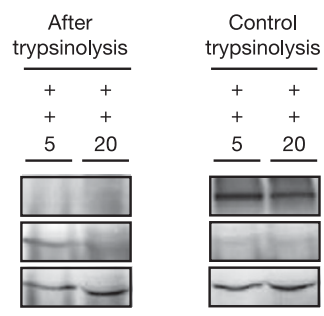

Figure 2 | MYPT-1 is the targeting subunit of merlin phosphatase. a, Co-immunoprecipitation (IP) of MYPT-1 and PP1 $\delta$ with merlin antibody (A-19). Immunoblots for MYPT-1 and PP1 1 . b, Pull-down of purified recombinant C-merlin with GST-C-MYPT-1. c, Co-immunoprecipitations of wild-type and mutant merlin (S518A or S518D) with MYPT-1 from cell lysates. d, In vitro dephosphorylation of merlin by the MYPT-1-PP1 $\delta$ complex immunoprecipitated with anti-MYPT-1. e, Trypsinolysis of MYPT-1-PP1 $\delta$ strongly reduces the catalytic function (phospho-merlin added after trypsin inhibitor for the periods indicated).
PP1 $\delta$ towards merlin we performed trypsinolysis ${ }^{12}$. However, the degradation of MYPT-1 by trypsin, which releases a fully active PP1 $\delta$, decreased merlin dephosphorylation, indicating that MYPT-1 acts as a substrate specifier (Fig. 2e).

We determined by GST pull-downs (constructs in Fig. 3a) the interacting domains of both merlin and MYPT-1. Only C-merlin312-595, but not C-merlin-507-595 or C-merlin-342-595, precipitated MYPT-1 from lysates of RT4 cells (Fig. 3b). The interacting domain (residues 312-341) seems to be the amino-terminal part of the $\alpha$-helical coiled-coil region of merlin ${ }^{13}$, which is coarsely consistent with the MYPT-1-interacting region in moesin ${ }^{8}$. MYPT-1 binding was essential for merlin dephosphorylation in vivo because only C-merlin-342-595 could not be dephosphorylated on serum withdrawal (Fig. 3c, and Supplementary Fig. S3).

Dephosphorylation of merlin is important for its tumour suppressor function ${ }^{5,6}$. Several human missense mutations associated with neurofibromatosis type 2 have been found in the MYPT-1-interacting region of merlin ${ }^{14-17}$. To test whether these mutations affect the interaction with the phosphatase, we generated GST-C-merlin constructs carrying one of the naturally occurring mutations L316F, Q324L and L339F. One mutant, L339F, found in a patient with sporadic meningioma ${ }^{17}$, was indeed defective in MYPT-1 binding (Fig. 3d). a
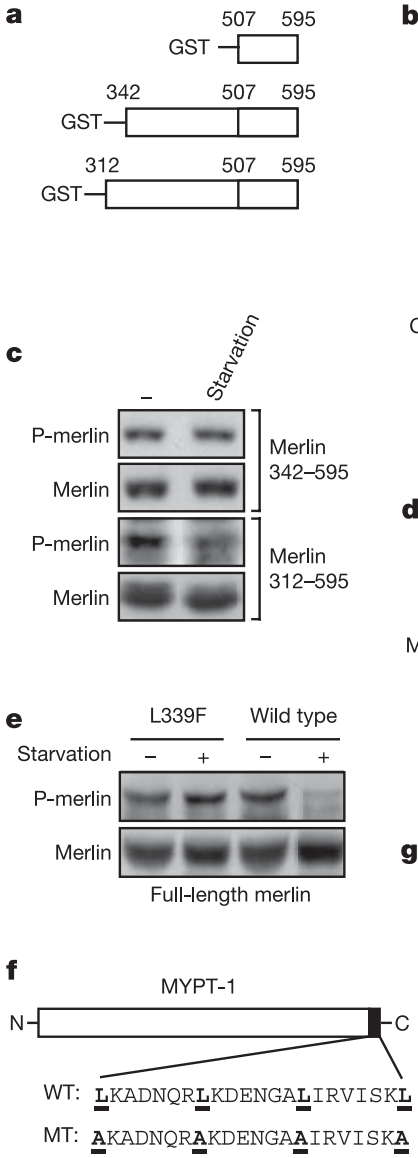

d

$\mathbf{g}$
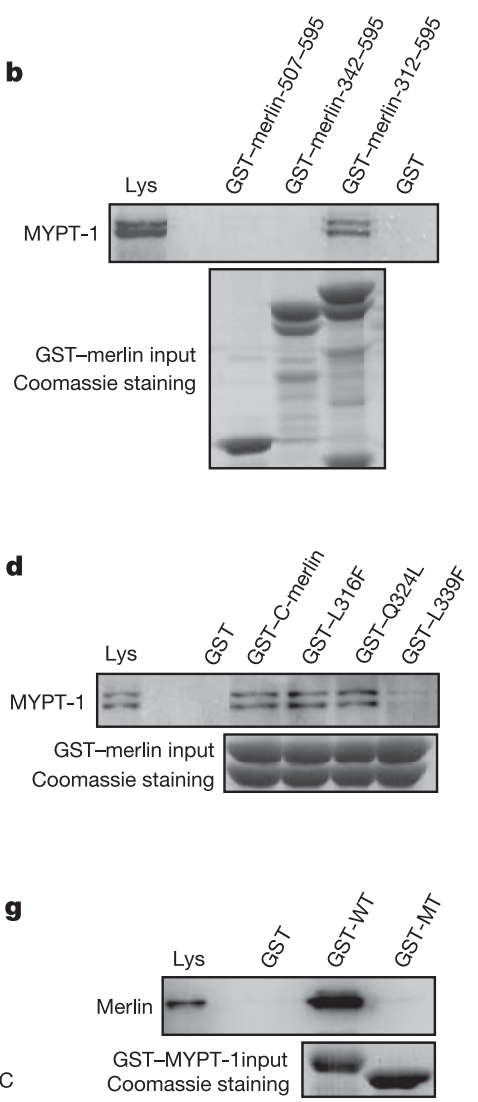

Figure 3 | Interaction domains of merlin and MYPT-1. a, GST-merlin constructs. b, GST-C-merlin deletion mutant pull-downs from cell lysates of MYPT-1 detected by immunoblot. $\mathrm{c}, \mathrm{C}$-merlin lacking the interaction region (amino-acid positions 312-341) is not dephosphorylated in vivo in response to serum withdrawal (immunoblot with phospho-merlin-specific antibodies). d, GST pull-downs of missense mutations in the MYPT-1 interacting region from a patient with neurofibromatosis type 2. e, The MYPT-1-binding-deficient L339F mutant does not dephosphorylate merlin in vivo. f, Scheme of MYPT-1 leucine zipper (LZ) wild type (WT) and mutant (MT). $\mathbf{g}$, The MYPT-1 leucine zipper mutation shown in $\mathbf{f}$ destroys the interaction with merlin (Protein stain; LZ and WT migrate differently.) 
The disruption of the interaction with the activating phosphatase and loss of the subsequent dephosphorylation of the L339F mutant protein (Fig. 3e) could well explain its tumour-promoting effect.

MYPT-1 has a leucine zipper domain at its $C$ terminus (Fig. 3f), which would be an ideal partner for the coiled-coil region of merlin. Mutation of the leucine residues to alanine (MYPT-1 L/A; 4) completely abolished MYPT-1 binding to other known substrates ${ }^{18-20}$. The GST-C-MYPT-1 L/A mutant, in contrast with the wild type, could not bind merlin (Fig. 3g). MYPT-1-associated PP1 $\delta$ therefore carries at least two binding sites for substrate, the $\mathrm{N}$ terminus for myosin ${ }^{9,10}$ and the C-terminal leucine zipper domain for merlin.

Phosphatase activities need to be regulated; for example, MYPT-1PP1 $\delta$ is inactivated by phosphorylation. Accordingly, MYPT-1 is dephosphorylated in the same way as merlin after serum withdrawal (Supplementary Fig. S4). In addition, MYPT-1-PP1ס is counterregulated by several cellular inhibitors ${ }^{10,21}$, namely phosphatase holoenzyme inhibitor $(\mathrm{PH}-1)^{22}$, kinase-enhanced phosphatase inhibitor (KEP-1 $)^{23}$ and the $17-\mathrm{kDa}$ protein kinase $\mathrm{C}$ potentiated inhibitor (CPI-17), the most specific and potent MYPT-1-PP1 $\delta$ inhibitor $^{10,24}$. Overexpression of CPI-17 in RT4tet-merlin cells prevented merlin S518 dephosphorylation induced by serum withdrawal (Fig. 4a). Thus, inhibition of MYPT-1-PP1 $\delta$ by CPI-17 and thus of merlin activation inhibited the suppression of anchorageindependent growth (Fig. 4b).

To study the CPI-17-mediated inhibition of merlin dephosphorylation in non-tumorigenic cells, we chose NIH 3T3 cells, which contain no detectable CPI-17. CPI-17 expression in NIH 3T3 cells increased the level of merlin phosphorylation (Fig. 4c, inset) and, as expected, that of moesin, a known substrate of MYPT-1-PP1 $\delta^{8}$ (data not shown). In addition, CPI-17 expression and subsequent inhibition of MYPT-1-PP1 1 , already known as an actomyosin contractility regulator, promoted cell shape changes and increased motility (data not shown). The expression of CPI-17 in NIH 3T3 cells caused all aspects of tumorigenic transformation: focus formation in the confluent monolayer culture and colony growth in soft agar (Supplementary Fig. S5a), and the formation of subcutaneous tumours when injected into nude mice (Fig. 4c, and Supplementary Fig. S5b). Because MYPT-1-PP1 $\delta$ acts on several other substrates besides merlin that could be responsible for tumour suppression, we attempted to rescue cells from CPI-17-induced transformation by expressing constitutively active merlin (S518A) under a doxycyclineinducible promoter. Merlin S518A, but not wild-type merlin, inhibited the growth of agar colonies despite overexpression of CPI-17 (Fig. 4d), indicating that MYPT-1-PP1 $\delta$ suppresses the tumorigenic phenotype by activating merlin. How the inhibition of MYPT-1-PP1 $\delta$ creates tumorigenic properties could be explained by knowing that merlin interferes with the activation of Ras (H.M., T.S. and P.H., unpublished observations); in agreement, inhibition of MAP kinase/ ERK kinase blocked CPI-17-dependent transformation: Supplementary Fig. S5c). Thus, expression of CPI-17 in NIH 3 T3 cells released Ras from inhibition by merlin and caused elevated levels of active Ras-GTP and phospho-ERK (Fig. 4e). This was prevented by introduction of the constitutively active S518A merlin mutant (Fig. 4f). The causal chain CPI-17-MYPT-1-merlin-Ras was further documented by downregulating MYPT-1 by short interfering RNA (siRNA), which also blocked merlin dephosphorylation and increased Ras activation (shown for HeLa cells in Fig. 4g; not shown for RPM-MC cells).

From gene disruption in the mouse $e^{2,3}$ we know that merlin is an essential gene. In addition, tumours in humans often carry merlin mutations ${ }^{25-27}$. On the basis of our data, elevated CPI-17 expression in tumorigenesis would be an alternative to loss of merlin function due to mutation. We found several human tumour cell lines with elevated CPI-17 mRNA (Fig. 4h, upper panel) and protein expression (Fig. 4h, lower panel). The abundance of CPI-17 protein in some tumour cell lines was almost as great as the ectopic expression level in both RT4tetmerlin/CPI-17 and NIH 3T3-CPI-17 cells (Fig. 4i, and not shown). To show that CPI-17 is involved in human tumorigenesis, we
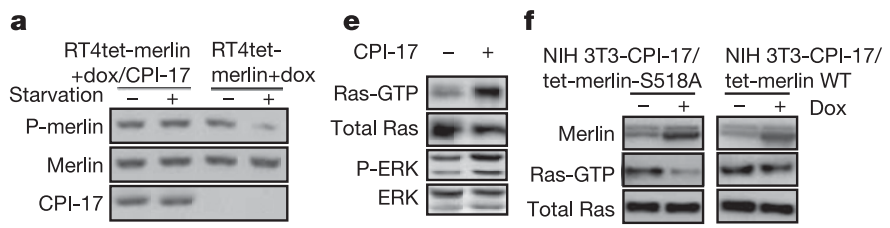

b
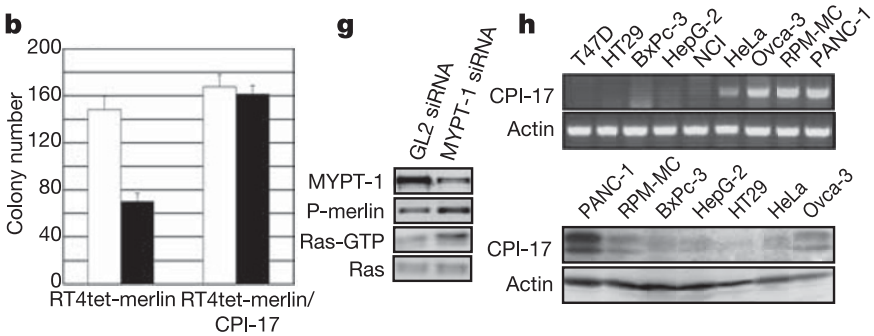

c
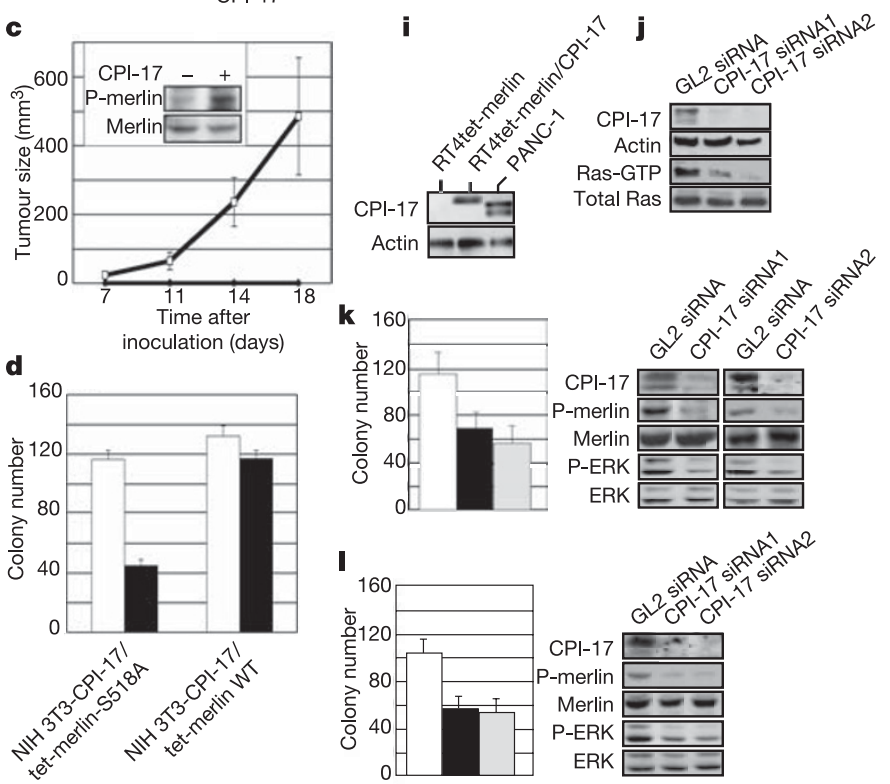

Figure 4 | The MYPT-1-PP1 $\delta$-specific inhibitor CPI-17 inhibits merlin activation, promotes Ras activation and induces cellular transformation. a, b, CPI-17 expression prevents merlin desphosphorylation on serum withdrawal (a; immunoblots for phospho-S518 merlin, CPI-17 and bulk merlin) and merlin-dependent reduction of colony growth on soft agar (b; means \pm s.d. of three independent experiments; $P<0.01$, Student's $t$-test). Open bars in $b$, no doxycycline; filled bars, with doxycycline. c, CPI-17 (open symbols) increases merlin phosphorylation (the inset shows an immunoblot for phospho-S518 merlin in NIH 3T3 cells) and transforms $\mathrm{NIH} 3 \mathrm{~T} 3$ cells as shown by tumour growth in nude mice. Filled symbols, NIH 3T3 cells. Results are means \pm s.e.m. of tumour volumes from four animals. d, f, CPI-17-promoted agar colony growth (d; means \pm s.d. of at least three independent experiments; $P<0.01$ ) and Ras activation (f; method as in e) is inhibited by doxycycline-induced mutant merlin S518A (which does not require dephosphorylation), but not the wild-type (WT) merlin. Open bars in d, no doxycycline; filled bars, with doxycycline. e, CPI-17 increases Ras activity in NIH 3T3 cells. Ras-GTP was precipitated with Raf-1RBD; immunoblots were performed with the antibodies indicated. g, MYPT-1 downregulation by siRNA increases merlin phosphorylation and promotes Ras activation in HeLa cells. $\mathbf{h}$, Expression of CPI-17 in different human tumour cell lines (upper panel, RT-PCR; lower panel, immunoblot). i, Comparison of ectopic Flag-tagged CPI-17 expression in RT4tet-merlin/CPI-17 cells with that in PANC-1 cells. j, siRNA downregulation of CPI-17 in RPM-MC cells decreases Ras activation. k, I, CPI-17 knockdown by two different siRNAs (black bars, siRNA1; grey bars, siRNA2) inhibits colony growth on agar and ERK activation in PANC-1 cells (k) and RPM-MC cells (I) compared with control luciferase (GL2; white bars). Quantitative results are means \pm s.d. of at least three independent experiments; $P<0.01$. Lysates were immunoblotted as indicated. 
downmodulated CPI-17 by siRNA in two human tumour cell lines, the pancreatic cell line PANC-1 and the melanoma cell line RPM-MC, and tested agar colony growth as a measure of the transformed phenotype. The colony number was indeed significantly decreased by two different CPI-17 specific siRNAs (Fig. 4k, 1). As expected, the CPI-17 siRNA decreased the level of merlin phosphorylation and consequently Ras and ERK activity in both tumour cell lines (Fig. 4j-1).

Our observations show that MYPT-1-PP1 $\delta$ is important for cellular growth control in that it activates the tumour suppressor protein merlin, which subsequently targets the Ras pathway. Transformation by the oncoprotein CPI-17 demonstrates that the elimination of PP1 activity shown here and that of $\mathrm{PP} 2 \mathrm{~A}^{28}$ both contribute to tumorigenesis. Although merlin inactivation is a major determinant of transformation by CPI-17, we also find elevated phosphorylation of ezrin, radixin and moesin (ERM; data not shown). Active phosphorylated ERM proteins are probably essential for Ras activation and proliferation control (H.M., T.S. and P.H., unpublished observations). Because merlin antagonizes ERM function ${ }^{5}$, CPI-17 probably induces a tumour-promoting activity of ERMs and loss of the counteracting role of merlin. Taken together, our data form the basis for proposing a new pathway leading to transformation that includes a phosphatase and an inhibitor involved in actomyosin contractility.

\section{METHODS}

Enzymes and other materials. These are described in Supplementary Information.

Antibodies and plasmids. Flag antibody (M2) was obtained from Sigma; phospho-ERK (Thr 202/Tyr 204) antibody from Cell Signalling; CPI-17 antibodies from Santa Cruz and Upstate; MYPT-1 antibodies from Santa Cruz for immunoprecipitations and Becton Dickinson for immunoblotting; phosphoS518-merlin antibody from Rockland; phospho-MYPT-1 (Thr 696), Erk1 (K23), merlin (C-18 and A-19), actin (I-19), pan PP1 (E-9), pan PP2A (C-20) and PP1ס (also called $\beta \mathrm{N}-9$ ) antibodies from Santa Cruz; and Ras antibody (Ras10) from Upstate. GST-C-MYPT-1-expressing vector (wild-type and mutated type) was a gift from H. K. Surks. CPI-17-expressing vector was provided by A. Aitken. EcoR1/ fragments encoding C-merlin cDNA (residues 299-595, 312-595, 342-595 and 507-595) were amplified from pUHD10-3-NF-2 and subcloned into pGEX 4T.1 to produce GST-C-merlin.

Cell culture. Cells were maintained in DMEM medium supplemented with 10\% FCS. Human tumour cell lines are described in Supplementary Information.

Transfections. Transfections were performed with Fugene 6 (Roche), in accordance with the manufacturer's instructions. The generation of stable clones is described in Supplementary Information.

siRNA. Sequences of siRNA are described in Supplementary Information. Gene silencing was achieved by transfection with $20 \mathrm{nM}$ siRNA by using Oligofectamine (Invitrogen) in accordance with the manufacturer's instructions.

SDS-PAGE, western blotting and Coomassie brilliant blue staining. Procedures are described in Supplementary Information.

RT-PCR. The procedure is described in Supplementary Information.

Immunoprecipitation and GST pull-down assay. The procedure is described in Supplementary Information.

In vitro dephosphorylation assay. The procedure is described in Supplementary Information.

Trypsinolysis of MYPT-1. Immunoprecipitated MYPT-1-PP1 $\delta$ complex was mixed with an equal volume of either $1 \mathrm{mg} \mathrm{ml}^{-1}$ trypsin (trypsinolysis) or $20 \mathrm{mg} \mathrm{ml}^{-1}$ soybean trypsin inhibitor (control trypsinolysis) and incubated for 5 min at $30^{\circ} \mathrm{C}$. Incubations were terminated with an equal volume of $20 \mathrm{mg} \mathrm{ml}^{-1}$ soybean trypsin inhibitor or $1 \mathrm{mg} \mathrm{ml}^{-1}$ trypsin, respectively. The merlin substrate was incubated at $30^{\circ} \mathrm{C}$ for $5-20 \mathrm{~min}$. Phosphorylated merlin was prepared as described in Supplementary Information.

Soft agar assay. The procedure is described in Supplementary Information. Tumour growth in vivo. The procedure is described in Supplementary Information.

\section{Received 21 October 2005; accepted 2 May 2006.}

1. McClatchey, A. I. Merlin and ERM proteins: unappreciated roles in cance development? Nature Rev. Cancer 3, 877-883 (2003).

2. McClatchey, A. I. et al. Mice heterozygous for a mutation at the Nf2 tumor suppressor locus develop a range of highly metastatic tumors. Genes Dev. 12 1121-1133 (1998)

3. McClatchey, A. I., Saotome, I., Ramesh, V., Gusella, J. F. \& Jacks, T. The Nf2 tumor suppressor gene product is essential for extraembryonic development immediately prior to gastrulation. Genes Dev. 11, 1253-1265 (1997).

4. Xiao, G. H., Chernoff, J. \& Testa, J. R. NF2: the wizardry of merlin. Genes Chromosom. Cancer 38, 389-399 (2003).

5. Morrison, $\mathrm{H}$. et al. The NF2 tumor suppressor gene product, merlin, mediates contact inhibition of growth through interactions with CD44. Genes Dev. 15 968-980 (2001)

6. Shaw, R. J. et al. The Nf2 tumor suppressor, merlin, functions in Rac-dependent signalling. Dev. Cell 1, 63-72 (2001)

7. Dounay, A. B. \& Forsyth, C. J. Okadaic acid: the archetypal serine/threonine protein phosphatase inhibitor. Curr. Med. Chem. 9, 1939-1980 (2002).

8. Fukata, Y. et al. Association of the myosin-binding subunit of myosin phosphatase and moesin: dual regulation of moesin phosphorylation by Rho-associated kinase and myosin phosphatase. J. Cell Biol. 141, 409-418 (1998).

9. Hartshorne, D. J. \& Hirano, K. Interactions of protein phosphatase type 1, with a focus on myosin phosphatase. Mol. Cell. Biochem. 190, 79-84 (1999).

10. Ito, M., Nakano, T., Erdodi, F. \& Hartshorne, D. J. Myosin phosphatase: structure, regulation and function. Mol. Cell. Biochem. 259, 197-209 (2004).

11. Beullens, M., Van Eynde, A., Stalmans, W. \& Bollen, M. The isolation of novel inhibitory polypeptides of protein phosphatase 1 from bovine thymus nuclei. J. Biol. Chem. 267, 16538-16544 (1992).

12. Colbran, R. J. et al. Association of brain protein phosphatase 1 with cytoskeletal targeting/regulatory subunits. J. Neurochem. 69, 920-929 (1997).

13. Rouleau, G. A. et al. Alteration in a new gene encoding a putative membraneorganizing protein causes neuro-fibromatosis type 2. Nature 363, 515-521 (1993).

14. Shimizu, T. et al. Structural basis for neurofibromatosis type 2. Crystal structure of the merlin FERM domain. J. Biol. Chem. 277, 10332-10336 (2002).

15. Baser, M. E. et al. Genotype-phenotype correlations for nervous system tumors in neurofibromatosis 2: a population-based study. Am. J. Hum. Genet. 75, 231-239 (2004).

16. Baser, M. E. et al. Presymptomatic diagnosis of neurofibromatosis 2 using linked genetic markers, neuroimaging, and ocular examinations. Neurology 47, 1269-1277 (1996).

17. De Vitis, L. R. et al. Screening for mutations in the neurofibromatosis type 2 (NF2) gene in sporadic meningiomas. Hum. Genet. 97, 632-637 (1996)

18. Surks, H. K., Richards, C. T. \& Mendelsohn, M. E. Myosin phosphatase-Rho interacting protein. A new member of the myosin phosphatase complex that directly binds RhoA. J. Biol. Chem. 278, 51484-51493 (2003).

19. Surks, H. K. \& Mendelsohn, M. E. Dimerization of cGMP-dependent protein kinase $1 \alpha$ and the myosin-binding subunit of myosin phosphatase: role of leucine zipper domains. Cell. Signal. 15, 937-944 (2003).

20. Surks, H. K. et al. Regulation of myosin phosphatase by a specific interaction with cGMP-dependent protein kinase l $\alpha$. Science 286, 1583-1587 (1999).

21. Feng, J. et al. Inhibitory phosphorylation site for Rho-associated kinase on smooth muscle myosin phosphatase. J. Biol. Chem. 274, 37385-37390 (1999).

22. Eto, M., Karginov, A. \& Brautigan, D. L. A novel phosphoprotein inhibitor of protein type-1 phosphatase holoenzymes. Biochemistry 38, 16952-16957 (1999).

23. Liu, Q. R. et al. KEPI, a PKC-dependent protein phosphatase 1 inhibitor regulated by morphine. J. Biol. Chem. 277, 13312-13320 (2002).

24. Eto, M., Senba, S., Morita, F. \& Yazawa, M. Molecular cloning of a nove phosphorylation-dependent inhibitory protein of protein phosphatase-1 (CPI17) in smooth muscle: its specific localization in smooth muscle. FEBS Lett. 410, 356-360 (1997).

25. Evans, D. G., Sainio, M. \& Baser, M. E. Neurofibromatosis type 2. J. Med. Genet. 37, 897-904 (2000)

26. Gutmann, D. H. New insights into the neurofibromatoses. Curr. Opin. Neurol. 7, 166-171 (1994).

27. Uppal, S. \& Coatesworth, A. P. Neurofibromatosis type 2. Int. J. Clin. Pract. 57, 698-703 (2003)

28. Hahn, W. C. \& Weinberg, R. A. Modelling the molecular circuitry of cancer. Nature Rev. Cancer 2, 331-341 (2002).

Supplementary Information accompanies the paper on www.nature.com/ nature.

Acknowledgements We thank N. Howells for the animal experiments performed in the Institute of Toxicology and Genetics at the Forschungszentrum Karlsruhe, and F. Böhmer and C. Calkhoven for helpful discussions. This work was supported by the Deutsche Forschungsgemeinschaft (DFG), the Association for International Cancer Research and the Sonderforschungsbereich (SFB).

Author Contributions H.M. was the project leader. H.M. and H.J. were responsible for the experimental and project design. H.J. performed most the experiments. H.M. and T.S. performed some of the experiments. H.M. and P.H. wrote the manuscript and all authors discussed the results and contributed to the writing of the manuscript. H.M. and T.S. prepared the final version of the manuscript.

Author Information Reprints and permissions information is available at npg.nature.com/reprintsandpermissions. The authors declare no competing financial interests. Correspondence and requests for materials should be addressed to H.M. (helen@fli-leibniz.de). 